\title{
Disoriented Chiral Condensates and Quantum Mechanical Isospin Correlation
}

\author{
Thomas D. Cohen, Manoj K. Banerjee, Marina Nielsen 1 and Xuemin Jin \\ Department of Physics and Center for Theoretical Physics \\ University of Maryland, College Park, MD 20742
}

March, 1994

\begin{abstract}
Classical considerations suggest that the probability distribution $P(R)$, where $R$ is the ratio of neutral pions to total pions emitted from a disoriented chiral condensate (which has been hypothesized to form in heavy ion reactions) is $R^{-1 / 2} / 2$. Quantum mechanical isospin correlations between the condensate and the remainder of the system can alter this. Moments of the $\mathrm{P}(\mathrm{R})$ distribution can be expressed in terms of expectation values of $\left(I^{2} / N^{2}\right)^{m}$ where $I$ is the isospin carried by the condensate, $N$ is the number of pions emitted and $\mathrm{m}$ is an arbitrary integer. We find that the probability distribution is very similar to the classical distribution for $0.1<R<0.9$ unless the isospin carried by the condensate is very large.
\end{abstract}

${ }^{1}$ Permanent address: Instituto de Física, Universidade de São Paulo, 01498 - SP- Brazil. 
Recently, there has been considerable attention given to the possible formation of a disoriented chiral condensate $(\mathrm{D} \chi \mathrm{C})$ in high energy collisions of heavy ions [1, 2, 3, , 4, 5, 6, 7, 8, 9]. The basic idea is rather simple. During the collision a thermalized region at high temperature may form. If the temperature is above the chiral restoration temperature, the chiral order parameters $\langle\bar{q} q\rangle$ and $\left\langle i \bar{q} \gamma_{5} \tau q\right\rangle$ will be zero in this region. If the system subsequently cools rapidly, the chiral-restored state becomes unstable. The notion of the disoriented chiral condensate concerns the response of the system to this instability. Clearly the system will eventually relax to its vacuum value in which $\langle\bar{q} q\rangle=\langle\bar{q} q\rangle_{\text {vac }} \approx-m_{\pi}^{2} f_{\pi}^{2} / m_{q}$, and $\left\langle i \bar{q} \gamma_{5} \tau q\right\rangle=0$ with the energy being radiated away by hadron emission.

One scenario for this relaxation involves two time scales: a relatively quick scale in which a large region settles into a disoriented chiral condensate and then a slower scale in which this $\mathrm{D} \chi \mathrm{C}$ relaxes to the physical vacuum. The $\mathrm{D} \chi \mathrm{C}$ is a region which corresponds to a chirally rotated vacuum - i.e., a region in which the magnitude of the chiral symmetry breaking is the same as in the vacuum but in which the symmetry breaking is not aligned in the $\langle\bar{q} q\rangle$ direction. The plausibility of this scenario stems from the following fact: apart from explicit chiral symmetry breaking (which is proportional to the current quark masses and, thus, small) and surface terms associated with the mismatch of the chiral direction in the condensate and the true vacuum (which for a large spatial volume of condensate constitutes a small fraction of the energy driving the system) all directions in chiral space are equally likely. The idea is that these effects which give a preference for "falling" in the $\langle\bar{q} q\rangle$ direction are small perturbations on the scale of the dynamical instability and hence the system is almost as likely to fall in an arbitrary direction in chiral space. Of course, it is clear that if a region of $\mathrm{D} \chi \mathrm{C}$ forms it will eventually decay to the physical vacuum due to the quark mass and surface effects.

Whether or not this scenario is, in fact, viable is a subject of current interest [8, 9, [10, 11]. The key question is whether or not large regions of $\mathrm{D} \chi \mathrm{C}$ form. Unfortunately, the detailed dynamics of heavy ion physics is far beyond what can be calculated directly from QCD and 
thus, studies of this kind are, of necessity based on simplified models such as the linear sigma model [12].

If a large volume of $\mathrm{D} \chi \mathrm{C}$ is formed, it will presumably decay via the emission of hadrons with a momentum of order $V^{-1 / 3}$ where $V$ is the volume of the region with a $\mathrm{D} \chi \mathrm{C}$. Being the lightest and able to carry off chirality pions are the most likely candidates for these hadrons. Thus, one expects an anomalously large number of low momentum pions in the rest frame of $\mathrm{D} \chi \mathrm{C}$. A second signature concerns the relative distribution of neutral pions .

If the low momentum pions were emitted statistically, with the pions uncorrelated with each other, then one would expect that the ratio $R=N_{0} / N$, of low momentum neutral pions to the total number of low momentum pions should have a probability distribution given by a binomial distribution. As $N \rightarrow \infty$ the distribution becomes sharply peaked at the average value, $\langle R\rangle=1 / 3$ with a variance $\left\langle R^{2}\right\rangle-\langle R\rangle^{2}=\frac{2}{9 N}$ which goes to zero.

It is often asserted [3, 4, 8], on the basis of a simple classical argument, that if the low mass pions were to come entirely from a $\mathrm{D} \chi C$, then the following nonstatistical probability distribution emerges:

$$
P(R)=\frac{1}{2 R^{1 / 2}}
$$

The two are so radically different that it should be easy to tell which describes the pion momentum distribution better.

The derivation of eq. (11) is essentially classical [1, 2]. The question we wish to address here is whether or not this simple form for $P(R)$ survives a quantum treatment. It has been pointed out previously [6, 7] that if the low momentum pions in the central rapidity region produced during the collision from the $\mathrm{D} \chi \mathrm{C}$ are in a quantum state of zero isospin, then the distribution in eq. (1) will be automatically reproduced in the large $N$ limit. However, there is no guarantee that the low momentum pions will carry zero isospin. Indeed, in a typical ultrarelativistic heavy ion reaction one would expect that even if low momentum pions are created in large number the vast majority of particles are "high momentum" pions and other energetic particles. The total isospin of the system will be carried by both low momentum 
pions and the high momentum particles. While in the absence of photon emission the sum of these two isospins must equal the total isospin of the system, a priori there is no reason to assume that the low momentum pions must be in zero or any other pure isospin state. In order to determine how much isospin will be carried by the low momentum pions one requires a reliable dynamical theory of the collision process leading to emission of low momentum pions. Such a calculation is well beyond the current state of the art. Instead, we will simply cast the final state in a useful form and draw phenomenological conclusions.

Before proceeding with our calculation it is probably useful to discuss briefly the general question of how the notion of a disoriented chiral condensate can be made sensible in a quantum mechanical context. One obvious point is that the condensed state is not the entire quantum mechanical state. Apart from the condensate, if formed, one expects a large number of statistically emitted "high energy" particles in a relativistic heavy ion reaction. Thus, the simplest possible description of the state would be as a product of a condensed state and a state describing the rest of the system. In fact, however, this is not viable. In general, if the chiral condensate is disoriented (i.e., not pointing in the $\sigma$ direction in chiral space) it points in some direction in isospin space, breaking isospin symmetry. We know, however, that the true quantum state does not break isospin (if we neglect quark mass differences and electromagnetic effects). Thus, the way to incorporate the classical notion of the disoriented chiral condensate into a quantum picture is as follows: the physical state, to good approximation, may be regarded as a superposition of product states (between a condensed state pointing in one chiral direction and the rest of the system). This superposition involves a sum over states with the condensate pointing in every chiral direction with some weighting function. This notion of obtaining a quantum mechanically meaningful state from a mean field state which breaks a symmetry is quite standard. For example, in nuclear structure physics one may encounter a Hartree-Fock solution which is deformed, i.e., which break rotational symmetry. The physical states, with good quantum numbers, are obtained by projection which involves superposing deformed states pointing in different directions. 
At this point, it is convenient to make a few simplifications. First, we consider collisions of two heavy ions of zero isospin. Second, we assume that the total isospin is conserved during the collision, i.e., we ignore radiative processes. Third, we assume that in such collisions pions are produced with essentially zero momentum in the rest frame of the condensate. This is possible only if the region occupied by these pions is very large. We consider events in which the number of zero momentum pions is large assuming that such events occur during heavy ion collisions.

The concept of a disoriented chiral condensate aligned in a nontrivial isospin direction is meaningful only if essentially all of the pions in the condensate will be aligned in the same isospin direction in the condensate. This trivially implies that the pions in the condensate are in a completely symmetric state in isospin and by Bose symmetry they must be in a symmetric spatial state. All of the physical consequences discussed in this letter depend essentially on the low momentum pions being in an isospin symmetric state. We should note that the signatures discussed in this letter can tell us only whether a state of low momentum pions is symmetric in isospin. We have no way to determine whether such a state has its origin in a disoriented chiral condensate or via some other (as yet unknown) mechanism. We will refer to any state containing only low momentum pions in an isospin symmetric state as a condensate regardless of the actual mechanism by which it is formed.

We will study moments of the probability distribution because it is possible to obtain compact analytical expressions for these quantities in the limit of large $N$. Calculation of $P(R)$ as a function of $R$ involves differences of large numbers and becomes rather unwieldy for $N>100$. We will show only a few illustrative results for $N=100$.

To begin, we group the particles in the final state into two classes, low momentum pions and everything else. Assuming that the low momentum pions are all zero momentum we see that one completely labels the state by three quantum numbers which can be either the numbers of $\pi^{ \pm}$and $\pi^{0}$, or, the total number of pions $N$, the total isospin $I_{c}$, and the third component of the isospin $i_{3}$. We write the most general final state with zero isospin in the 
form:

$$
|\psi ; I=0\rangle=\sum_{N, I_{c}, i_{3}} C_{N, I_{c}} \frac{(-)^{i_{3}}}{\sqrt{2 I_{c}+1}}\left|N, I_{c}, i_{3}\right\rangle_{\mathrm{low}}\left|\psi_{N} ; I_{c},-i_{3}\right\rangle_{\mathrm{high}},
$$

where $C_{N, I_{c}}$ is a numerical coefficient, the subscript "low" labels the states of the condensate containing low momentum pions. The notation $\left|\psi_{N} ; I_{c},-i_{3}\right\rangle_{\text {high }}$ denotes the state containing no low momentum pions and carrying isospin $I_{c}$ and third component of isospin $-i_{3}$; the subscript $N$ merely indicates that it is the state coupling to a state of $N$ low momentum pions. If all states are normalized one has the constraint that

$$
\sum_{N, I_{c}}\left|C_{N, I_{c}}\right|^{2}=1
$$

Quantum mechanical correlations between the isospin carried by the low momentum pions and the rest of the system are exhibited by eq. (2).

If all the low momentum pions are zero momentum, the low momentum pion states $\left|N, I_{c}, i_{3}\right\rangle_{\text {low }}$ may be generated from vacuum by application of the creation operators for the three types of pions. Here, it is convenient to work in a cartesian basis for the isospin and thus the creation operators are $a_{i}^{\dagger}$ with $i=1,2,3$. Given the assumption of only zero momentum pions, the normalized low momentum states of fixed $N, I_{c}$ and $i_{3}$ are unique

$$
\left|N, I_{c}, i_{3}\right\rangle_{\text {low }}=\int \mathrm{d} \Omega \mathrm{Y}_{I_{c}, i_{3}}(\Omega)(N !)^{-1 / 2}\left(\hat{n}(\Omega) \cdot a^{\dagger}\right)^{N}|v a c\rangle
$$

where $\hat{n}(\Omega)$ is a unit vector in the $\Omega$ direction and $\mathrm{Y}_{I_{c}, i_{3}}(\Omega)$ is the spherical harmonic function.

Consider an operator, $\mathcal{O}_{\text {low }}$ which acts only in the space of the low momentum pions, i.e., an operator whose matrix elements do not depend on $\left|\psi_{N} ; I_{c},-i_{3}\right\rangle_{\text {high }}$ at all. Since, the operator is unity in the "high" space, the expectation value of such an operator is simple:

$$
\left\langle\mathcal{O}_{\text {low }}\right\rangle=\sum_{N, I_{c}}\left|C_{N, I_{c}}\right|^{2} \widetilde{\mathcal{O}_{\text {low }}}
$$

where

$$
\begin{aligned}
\widetilde{\mathcal{O}}_{\text {low }}\left(I_{c}\right) & \equiv \sum_{i_{3}} \frac{1}{2 I_{c}+1}\left\langle N, I_{c}, i_{3}\left|\mathcal{O}_{\text {low }}\right| N, I_{c}, i_{3}\right\rangle_{\text {low }} \\
& =\sum_{i_{3}} \frac{1}{2 I_{c}+1}\left\langle N, I_{c}, i_{3}\left|\mathcal{O}_{\text {low }}^{\text {isoscalar part }}\right| N, I_{c}, i_{3}\right\rangle_{\text {low }}
\end{aligned}
$$




\begin{tabular}{|l|l|l|l|l|}
\hline \hline Moment & 1 & $\left\langle\frac{I^{2}}{N^{2}}\right\rangle$ & $\left\langle\frac{I^{4}}{N^{4}}\right\rangle$ & $\left\langle\frac{I^{6}}{N^{6}}\right\rangle$ \\
\hline$\langle R\rangle$ & $\frac{1}{3}$ & & & \\
\hline$\left\langle R^{2}\right\rangle$ & $\frac{1}{5}$ & $-\frac{1}{15}$ & & \\
\hline$\left\langle R^{3}\right\rangle$ & $\frac{1}{7}$ & $-\frac{3}{35}$ & & \\
\hline$\left\langle R^{4}\right\rangle$ & $\frac{1}{9}$ & $-\frac{2}{21}$ & $\frac{1}{105}$ & \\
\hline$\left\langle R^{5}\right\rangle$ & $\frac{1}{11}$ & $-\frac{10}{99}$ & $\frac{5}{231}$ & \\
\hline$\left\langle R^{6}\right\rangle$ & $\frac{1}{13}$ & $-\frac{15}{143}$ & $\frac{5}{143}$ & $-\frac{5}{3003}$ \\
\hline
\end{tabular}

Table 1: The coefficients $b_{i}^{m}$ defined in eq. (8) .

Note that the number of low momentum pions $N$, the number of low momentum neutral pions $N_{0}$, and the ratio $R=N_{0} / N$ are all examples of operators of the form of $\mathcal{O}_{\text {low }}$. For $\mathcal{O}_{\text {low }}=R^{m}$, the problem of calculating the moments of the distribution is reduced to evaluating matrix elements $\widetilde{R^{m}}$. The general result is given below:

$$
\lim _{N \rightarrow \infty} \widetilde{R^{m}}=\sum_{i=0}^{[m / 2]} \sum_{j=0}^{i}\left\{\frac{(-1)^{i+j} m !}{2^{2 i} i ! j !(m-2 i) !(i-j) ![2 j+2(m-i)+1]}\left(\frac{I_{c}\left(I_{c}+1\right)}{N^{2}}\right)^{i}\right\} .
$$

It is straightforward to obtain expressions for $\left\langle R^{m}\right\rangle$ in the limit $N \rightarrow \infty$ by using eqs. (5) and (17) and the obvious definition, $\left\langle I^{2} / N^{2}\right\rangle=\sum_{N, I_{c}}\left|C_{N, I_{c}}\right|^{2} I_{c}\left(I_{c}+1\right) / N^{2}$. Writing

$$
\lim _{N \rightarrow \infty}\left\langle R^{m}\right\rangle=\sum_{i=0}^{[m / 2]} b_{i}^{m}\left\langle\frac{I^{2 i}}{N^{2 i}}\right\rangle
$$

the results for the coefficients $b_{i}^{m}$ for $m=1$ through 6 are given in Table 1 . We note that these results agree with the classical result when $\left\langle\frac{I^{2}}{N^{2}}\right\rangle \ll 1$.

One sees from Table 1 that when $\left\langle\frac{I^{2}}{N^{2}}\right\rangle$ increases from 0 to 0.1 that moments of order 4 and higher change by as much as $\sim 10 \%$. Thus an experimental determination of high moments with sufficiently good accuracy can provide a measure of the isospin content of the condensate.

Fortunately, there is a much more promising experimental way of relating $\left\langle\frac{I^{2}}{N^{2}}\right\rangle$ to observable quantities. It has been noted by Greiner, Gong and Müller [7] that, if the low 
momentum pions come from an $I_{c}=0$ condensate then in every event the number of low momentum $\pi^{+}$'s must exactly equal the number of $\pi^{-}$'s. Conversely, difference between the two numbers provides a direct measure of the isospin content:

$$
\lim _{N \rightarrow \infty}\left\langle\frac{\left(N_{+}-N_{-}\right)^{2 i}}{N^{2 i}}\right\rangle=\frac{1}{2 i+1}\left\langle\frac{I^{2 i}}{N^{2 i}}\right\rangle .
$$

It is easy to derive the relation by noting that $N_{+}-N_{-}=i_{3}$ and using eqs. (5) and (6). Thus a measurement of $\left\langle\frac{\left(N_{+}-N_{-}\right)^{2}}{N^{2}}\right\rangle$ is the most direct and natural way to measure the isospin content of the condensate. In particular, the variance is given by

$$
\left\langle R^{2}\right\rangle-\langle R\rangle^{2}=\frac{4}{45}-\left\langle\frac{\left(N_{+}-N_{-}\right)^{2}}{5 N^{2}}\right\rangle .
$$

The result in eq. (10) is in a real sense model independent. It does, however, depend rather strongly on two underlying assumptions. The first is the assumption that a single large region of $\mathrm{D} \chi \mathrm{C}$ is formed. If multiple regions form with different orientations the results will certainly change, and the variance in the $R$ distribution will certainly be reduced. One might hope to use eq. (10) to experimentally test whether a single region of $\mathrm{D} \chi \mathrm{C}$ is, in fact produced. The second important assumption is that the total system (or at least the part of the system which ultimately contributes) is isospin zero. This will certainly be violated to a certain degree. The initial state may well not be isospin zero. More importantly, photons will be emitted during the heavy ion reaction which violate isospin conservation. It is essential to estimate how large an effect these isospin violating effects will have on the final results. Unfortunately, estimates of the corrections to eq. (10) due to isospin violation will, of necessity be model dependent.

We have discussed the effect of quantum mechanical isospin correlations on moments of the $P(R)$ distribution. It should be remembered, of course, that the moments are derived quantities - the primary quanity is $P(R)$ itself. We would like to understand the effect of isospin correlations on $P(R)$. We write the probability distribution as

$$
P(R)=\sum_{N, I_{c}}\left|C_{N, I_{c}}\right|^{2} P_{I_{c}}(R)
$$


where the probability of finding $N_{0}$ neutral pions in the state $\left|N, I_{c}, i_{3}\right\rangle_{\text {low }}$ is given by

$$
P_{I_{c}}(R)=\frac{1}{2 I_{c}+1} \sum_{i_{3}}\left|\left\langle N, I_{c}, i_{3} \mid N, I_{c}, N_{0}\right\rangle_{\text {low }}\right|^{2} .
$$

Following Horn and Silver [13], this probability is evaluated to be

$$
\begin{gathered}
P_{I_{c}}(R)=\frac{1}{2 I_{c}+1} \sum_{i_{3}} N_{0} !\left(\frac{N-N_{0}+i_{3}}{2}\right) !\left(\frac{N-N_{0}-i_{3}}{2}\right) ! \frac{\left(2 I_{c}+1\right) ! !\left(N-I_{c}\right) ! !}{\left(N+I_{c}+1\right) ! !} \times \\
\frac{2^{I_{c}-i_{3}-N_{0}}}{\sum_{j} \frac{2^{-2 j}}{\left(i_{3}+j\right) ! j !\left(I_{c}-i_{3}-2 j\right) !}}\left(\sum_{k} \frac{(-1)^{k} 2^{-2 k}}{k !\left(I_{c}+k\right) !\left(I_{c}-i_{3}-2 k\right) !\left(\frac{N-N_{0}-i_{3}-2 k}{2}\right) !\left(\frac{N_{0}+i_{3}-I_{c}+2 k}{2}\right) !}\right)^{2},
\end{gathered}
$$

where the sums are over all integers $i_{3}, j$ and $k$ such that the factorials can be defined.

We see clearly that, the quantum mechanical isospin correlations will also affect the probability distribution. Needless to say, we cannot calculate the distribution without a knowledge of the coefficients $C_{N, I_{c}}$, which, in turn, requires a knowledge of the reaction mechanism. However, we can learn something by examining $P_{I_{c}}(R)$ for a range of values of $I_{c}$. These probability distributions have sharp odd-even variations. Such variations will never be seen in an experiment where one measures $P(R)$. The sum over $I_{c}$ in eq. (11) smoothes out the variations. In addition, there is bound to be additional averaging over neighboring $N_{0}$ and $N$ because of the difficulty of counting the pions exactly. We suppress these oscillations partially by averaging over two neighboring numbers of neutral pions. We believe that the procedure improves markedly the appearance of the graphs without affecting the conclusions we draw. In Fig. 1 we show these partially averaged $P_{I_{c}}(R)$ vs. $R$ for $I_{c}=0,10,20,30$ and 60 for $N=100$. The distributions for $I_{c}=10$ and 20 are not distinguishable from the $I_{c}=0$ distribution which, one may recall, agrees with the classical distribution [14]. The distribution for $I_{c}=30$ is barely distinguishable. These graphs tell us that the classical result of eq.(四) is robust (except at the endpoint regions near $R=0$ and $R=1$ ) and will describe the actual distribution $P(R)$ unless there is massive isospin mixing. Conversely, agreement with the classical result will tell us little about the isospin content of the condensate. It will establish that the pions in the condensate are in fully isosymmetric state and, hence, in a fully space symmetric state. Information about the isospin admixtures is best obtained by 
studying the charge asymmetry distribution.

T.D.C. thanks Berndt Müller for introducing him to the problem and for helpful discussions. T.D.C., M.K.B., and X.J. thank the US Department of Energy for support of this research through grant No. DE-FG02-93ER-40762. T.D.C. and X.J. also acknowledge the support of the US National Science Foundations Presidential Young Investigator program through grant No. PHY-9058487. M.N. acknowledges the warm hospitality and congenial atmosphere provided by the Nuclear Theory Group of the University of Maryland and support from FAPESP-Brazil . 


\section{References}

[1] A. A. Anselm, Phys. Lett. B217 (1989) 169.

[2] A. A. Anselm and M. C. Ryskin Phys. Lett. B266 (1991) 482.

[3] J-P. Blaizot and A Krzywicki, Phys. Rev. D 46 (1992) 246.

[4] J. D. Bjorken, Acta. Physica Polonica B23 (1992) 637.

[5] K. L. Kowalski and C. C. Taylor, preprint CWRUTH-92-6 (1992).

[6] J. D. Bjorken, K. L. Kowalski and C. C. Taylor, preprint SLAC-PUB-6109 (1993).

[7] C. Greiner, C. Gong and B. Müller, Phys. Lett. B316 (1993) 226.

[8] , K. Rajagopal and F. Wilczek, Nucl. Phys. B379 (1993) 395; B404 (1993) 577.

[9] S. Gavin, A. Gocksch, R. D. Pisarski, preprint BNL-GGP-1(1993).

[10] J-P. Blaizot and A Krzywicki, preprint LPTHE Orsay 94/18.

[11] D. Boyanovsky, H.J. de Vega and R. Holmar, preprint LPTHE 94/01.

[12] M. Gell-Mann and M. Lévy, Nuovo Cimento 16, 705(1960).

[13] D. Horn and R. Silver, Ann. Phys. 66 (1971) 509.

[14] To be precise $P_{\text {classical }}=\frac{1}{2} P_{I_{c}=0}(R)$ for even $N_{0}$ only, the latter being zero for odd $N_{0}$. Here the factor of $\frac{1}{2}$ gets included through the averaging over neighboring odd and even $N_{0} \mathrm{~S}$. 


\section{Figure Captions}

Figure 1. $P_{I_{c}}(R)$ vs. $R$ for $I_{c}=0,10,20,30$ and 60 for $\mathrm{N}=100$. The graphs for $I_{c}=10$ and 20 are not distinguishable from that for $I_{c}=0$ and are not labeled. Other graphs are labeled by their isospins. 
This figure "fig1-1.png" is available in "png" format from: http://arxiv.org/ps/hep-ph/9403273v1 